\title{
Simplified Forecasting by Polynomial Regression With Equally Spaced Values of the Independent Variable
}

\author{
By Jack Laderman and Julian D. Laderman
}

\begin{abstract}
A method is developed and tables are presented which yield polynomial least squares forecasts by computing the inner product of two $n$-component vectors, where $n$ is the number of observed points.
\end{abstract}

1. Introduction. In this paper, a method is developed which makes it easy to forecast by polynomial regression when the values of the independent variable are equally spaced. More specifically, one can obtain the forecast yielded by a least squares polynomial by simply computing an inner product. This method of forecasting by least squares is easier than by any of the other simplified methods which have appeared in the literature. Many methods [1] avoid solving the normal equations. Instead the least squares polynomial is obtained by first computing moments from the data and then obtaining coefficients of the least squares polynomial from linear functions of these moments. Tables which give the coefficients of the linear functions are provided in those papers. Then a forecast is obtained by evaluating the polynomial at the desired value of the independent variable. The method presented in this paper avoids the computation of moments, the solving of the normal equations, and the evaluation of the polynomial. The tables presented are applicable for polynomials up to the seventh degree for 2 to 20 points with equally spaced values of the independent variable.

2. Forecasting Technique. Without loss of generality, the values of the independent variable, $x$, are to be taken $1,2,3, \ldots, n$ and the corresponding values of the dependent variable are denoted by $y_{1}, y_{2}, y_{3}, \ldots, y_{n}$. The vector formed by these $y$ values will be denoted by $Y$. The coefficients of the $k$ th degree least squares polynomial,

$$
\hat{y}=a_{0}+a_{1} x+a_{2} x^{2}+\cdots+a_{k} x^{k},
$$

are usually obtained by solving the normal equations

$$
\left(X^{\prime} X\right) A=b \text {, }
$$

Received April 2, 1981; revised July 6, 1981.

1980 Mathematics Subject Classification. Primary 62J02; Secondary 65 U05.

Key words and phrases. Forecasting by least squares, finite differences, matrix inversion by the square root method. 
where

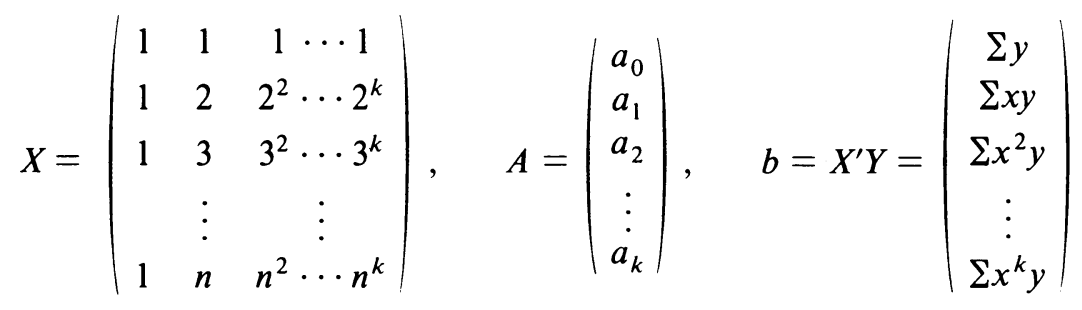

and $X^{\prime}$ is the transpose of $X$. Therefore

$$
A=\left(X^{\prime} X\right)^{-1} b \text {. }
$$

The solution for the $a_{j}$ given by (3) yields linear forms in the $y_{i}$, say $L_{j}(y)$. By replacing the $a_{j}$ in (1) by these linear forms, the $k$ th degree least squares polynomial, $\hat{y}$, can be expressed as

$$
\hat{y}=L_{0}(y)+L_{1}(y) x+L_{2}(y) x^{2}+\cdots+L_{k}(y) x^{k} .
$$

Then by letting $x=n+1$, and combining the $y_{i}$ terms in (4), the least squares forecast, $\hat{y}$, for $x=n+1$, becomes a linear form in $y_{i}$, say

$$
\hat{y}=c_{1} y_{1}+c_{2} y_{2}+c_{3} y_{3}+\cdots+c_{n} y_{n} \text {. }
$$

Tables are presented which contain the values of $c_{1}$ to $c_{n}$ for $(n, k)$ pairs, where $n=2,3,4, \ldots, 20$ and $k=1,2,3, \ldots, 7$ with $k \leqslant n-1$. The forecast for $x=n+1$ is simply the inner product $Y \cdot C$, where $C$ is the vector of the appropriate column of $c_{i}$ values listed in the table.

These tables can also be used to forecast $y_{n+2}, y_{n+3}$, etc. This can be done because the forecast for $y_{n+1}$ by a $k$ th degree least squares polynomial yields a value which lies on that $k$ th degree equation. Therefore, if a $k$ th degree polynomial forecast is desired for $y_{n+2}$ the forecast for $y_{n+1}$ lies on the least squares polynomial implicitly employed, and so this new point can be included with the others to forecast $y_{n+2}$. Thus, the values $y_{1}, y_{2}, y_{3}, \ldots, y_{n}, \hat{y}_{n+1}$ can be used with the $n+1$ values of $c_{i}$ taken from the $n+1$ table to forecast $y_{n+2}$. Continuing in this manner, least squares polynomial forecasts can be obtained for other $y_{i}$. Repeated use of this procedure for forecasting at an $x$ value far above $n$ will usually cause a build up of rounding errors. This is because the forecast at an $x$ value $j$ intervals above $n$ is obtained from the $n$ observed values of $y$ and from $j-1$ forecasted values.

3. Tables of $c_{i}$. To use the tables of $c_{i}$ values, first go to the table headed with the appropriate value of $n$. The $c_{i}$ values for forecasting with a $k$ th degree polynomial are in the column headed by that $k$ value. Then $\sum_{i=1}^{n} c_{i} y_{i}$ gives the forecast at $x=n+1$.

Example. Forecast the $y$ values by a second degree least squares polynomial at $x=8.5$ and at $x=9.0$ given the following 10 points of data:

\begin{tabular}{|r|r|r|r|r|r|r|r|r|r|r|}
\hline$x$ & 3.5 & 4.0 & 4.5 & 5.0 & 5.5 & 6.0 & 6.5 & 7.0 & 7.5 & 8.0 \\
\hline$y$ & 14.1 & 14.3 & 14.4 & 14.8 & 15.5 & 17.0 & 18.1 & 19.4 & 20.2 & 21.1 \\
\hline
\end{tabular}

The forecast for $x=8.5$ is obtained by using the $c_{i}$ values from Table 9 in the column headed by $k=2$. Then $\sum_{i=1}^{10} c_{i} y_{i}=23.1$ is the forecast. 
The forecast for $x=9.0$ is obtained by using the $c_{i}$ values from Table 10 ( $k=2$ column) including the 11th $y$ value of 23.1 at $x=8.5$. Then $\sum_{i=1}^{11} c_{i} y_{i}=24.7$ is the forecast.

To check the accuracy of the method, the second-degree least squares polynomial was obtained by using the Statistical Package for the Social Sciences (SPSS). The polynomial is

$$
\hat{y}=15.39727-1.378633 x+.2681814 x^{2} .
$$

The values obtained from this polynomial at $x=8.5$ and $x=9.0$ to three significant figures are 23.1 and 24.7 , respectively.

4. Remarks About the Tables. If all $y_{i}$ are set equal to a constant, $w$, the forecast for $y_{n+1}$ must be $w$. It then follows that the sum of the $c_{i}$ values for each $(n, k)$ pair is 1 .

When $k=0$, the least squares polynomial for any $n$ is the arithmetic mean of the $y_{i}$. For this value of $k$, all the $c_{i}$ are equal to $1 / n$, so these values are not listed in the tables.

For any $(n, k)$ pair in the tables, it is seen that the values of the $c_{i}$ lie on a $k$ th degree polynomial in $i$. It is conjectured that this property holds also for all other $(n, k)$ pairs for $k \leqslant n-1$. Under this assumption the values of $c_{i}$ for $k=n-1$ are signed binomial coefficients. This follows by first expressing the $(k+1)$ st differences [3] as

$$
\Delta^{k+1} y_{1}=\sum_{i=0}^{k+1}(-1)^{k-i+1}\left(\begin{array}{c}
k+1 \\
i
\end{array}\right) y_{i+1}
$$

Since the $(k+1)$ st differences of a $k$ th degree polynomial are zero, it follows that

$$
\sum_{i=0}^{n}(-1)^{n-i}\left(\begin{array}{c}
n \\
i
\end{array}\right) y_{i+1}=0 .
$$

Therefore

$$
y_{n+1}=\sum_{i=0}^{n-1}(-1)^{n-i-1}\left(\begin{array}{c}
n \\
i
\end{array}\right) y_{i+1} .
$$

5. Details of the Computation. The tables were computed at Herbert H. Lehman College using an Amdahl 470/V6 computer located at the Computer Center of the City University of New York. The programming language chosen was APL because this language is convenient for operations on matrices. The entries were rounded off to four decimal places. The program incorporated a check that the sum of the $c_{i}$ for each $(n, k)$ pair is 1 except for rounding.

The least accurate step in the computation of the table entries occurred in the process of obtaining the inverse of $X^{\prime} X$ when $k=7$. For this reason the inverse of $X^{\prime} X$, which is a symmetric matrix, was also obtained for these cases by programming appropriate steps called for in the square root method [2]. In that method, a triangular matrix, $S$, is obtained such that $X^{\prime} X=S^{\prime} S$. Since $\left(X^{\prime} X\right)^{-1}=S^{-1}\left(S^{\prime}\right)^{-1}$, this last expression was used to obtain $\left(X^{\prime} X\right)^{-1}$. The $c_{i}$ values resulting from this revised program agreed with those obtained from the original program when rounded off to four decimal places. 
Since the $c_{i}$ values for any $(n, k)$ pair in the tables satisfy a $k$ th degree polynomial, the entries were checked by differencing to make sure the $(k+1)$ st differences are zero. This was done for all $(n, k)$ pairs for $n \geqslant k+2$.

TABLE 1

Values of $c_{i}$ for $n=2$

\begin{tabular}{|c|c|}
\hline$i$ & $k=1$ \\
\hline 1 & -1.0000 \\
2 & 2.0000 \\
\hline
\end{tabular}

TABLE 2

Values of $c_{i}$ for $n=3$

\begin{tabular}{|r|r|r|}
\hline $\mathrm{i}$ & $\mathrm{k}=1$ & \multicolumn{1}{c|}{$k=2$} \\
\hline 1 & -.6667 & 1.0000 \\
2 & .3333 & -3.0000 \\
3 & 1.3333 & 3.0000 \\
\hline
\end{tabular}

TABLE 3

Values of $c_{i}$ for $n=4$

\begin{tabular}{|l|c|r|r|}
\hline$i$ & $k=1$ & $k=2$ & \multicolumn{1}{c|}{$k=3$} \\
\hline 1 & -.5000 & .7500 & -1.0000 \\
2 & .0000 & -1.2500 & 4.0000 \\
3 & .5000 & -.7500 & -6.0000 \\
4 & 1.0000 & 2.2500 & 4.0000 \\
\hline
\end{tabular}

TABLE 4

Values of $c_{i}$ for $n=5$

\begin{tabular}{|r|c|r|r|r|}
\hline $\mathrm{i}$ & $\mathrm{k}=1$ & $\mathrm{k}=2$ & \multicolumn{1}{c|}{$\mathrm{k}=3$} & \multicolumn{1}{c|}{$\mathrm{k}=4$} \\
\hline 1 & -.4000 & .6000 & -.8000 & 1.0000 \\
2 & -.1000 & -.6000 & 2.2000 & -5.0000 \\
3 & .2000 & -.8000 & -.8000 & 10.0000 \\
4 & .5000 & .0000 & -2.8000 & -10.0000 \\
5 & .8000 & 1.8000 & 3.2000 & 5.0000 \\
\hline
\end{tabular}

TABLE 5

Values of $c_{i}$ for $n=6$

\begin{tabular}{|l|c|c|r|r|r|}
\hline$i$ & $k=1$ & $k=2$ & $k=3$ & \multicolumn{1}{c|}{$k=4$} & \multicolumn{1}{c|}{$k=5$} \\
\hline 1 & -.3333 & .5000 & -.6667 & .8333 & -1.0000 \\
2 & -.1333 & -.3000 & 1.3333 & -3.1667 & 6.0000 \\
3 & .0667 & -.6000 & .3333 & 3.3333 & -15.0000 \\
4 & .2667 & -.4000 & -1.3333 & 1.6667 & 20.0000 \\
5 & .4667 & .3000 & -1.3333 & -5.8333 & -15.0000 \\
6 & .6667 & 1.5000 & 2.6667 & 4.1667 & 6.0000 \\
\hline
\end{tabular}


TABLE 6

Values of $c_{i}$ for $n=7$

\begin{tabular}{|r|c|r|r|r|r|r|}
\hline$i$ & $k=1$ & $k=2$ & \multicolumn{1}{c|}{$k=3$} & $k=4$ & \multicolumn{1}{c|}{$k=5$} & \multicolumn{1}{c|}{$k=6$} \\
\hline 1 & -.2857 & .4286 & -.5714 & .7143 & -.8571 & 1.0000 \\
2 & -.1429 & -.1429 & .8571 & -2.1429 & 4.1429 & -7.0000 \\
3 & .0000 & -.4286 & .5714 & 1.0000 & -6.8571 & 21.0000 \\
4 & .1429 & -.4286 & -.4286 & 2.1429 & 2.1429 & -35.0000 \\
5 & .2857 & -.1429 & -1.1429 & -.7143 & 7.1429 & 35.0000 \\
6 & .4286 & .4286 & -.5714 & -3.5714 & -9.8571 & -21.0000 \\
7 & .5714 & 1.2857 & 2.2857 & 3.5714 & 5.1429 & 7.0000 \\
\hline
\end{tabular}

TABLE 7

Values of $c_{i}$ for $n=8$

\begin{tabular}{|c|c|r|r|r|r|r|r|}
\hline$i$ & $k=1$ & $k=2$ & $k=3$ & $k=4$ & $k=5$ & $k=6$ & \multicolumn{1}{c|}{$k=7$} \\
\hline 1 & -.2500 & .3750 & -.5000 & .6250 & -.7500 & .8750 & -1.0000 \\
2 & -.1429 & -.0536 & .5714 & -1.5179 & 3.0000 & -5.1250 & 8.0000 \\
3 & -.0357 & -.3036 & .5714 & .0893 & -3.2500 & 11.3750 & -28.0000 \\
4 & .0714 & -.3750 & .0000 & 1.4464 & -1.5000 & -9.6250 & 56.0000 \\
5 & .1786 & -.2679 & -.6429 & .8036 & 3.7500 & -4.3750 & -70.0000 \\
6 & .2857 & .0179 & -.8571 & -1.3393 & 2.0000 & 16.6250 & 56.0000 \\
7 & .3929 & .4821 & -.1429 & -2.2321 & -6.7500 & -14.8750 & -28.0000 \\
8 & .5000 & 1.1250 & 2.0000 & 3.1250 & 4.5000 & 6.1250 & 8.0000 \\
\hline
\end{tabular}

TABLE 8

Values of $c_{i}$ for $n=9$

\begin{tabular}{|r|r|r|r|r|r|r|r|}
\hline$i$ & $k=1$ & $k=2$ & $k=3$ & $k=4$ & $k=5$ & \multicolumn{1}{c|}{$k=6$} & \multicolumn{1}{c|}{$k=7$} \\
\hline 1 & -.2222 & .3333 & -.4444 & .5556 & -.6667 & .7778 & -.8889 \\
2 & -.1389 & .0000 & .3889 & -1.1111 & 2.2500 & -3.8889 & 6.1111 \\
3 & -.0556 & -.2143 & .5079 & -.2778 & -1.5000 & 6.4444 & -16.8889 \\
4 & .0278 & -.3095 & .1905 & .8333 & -1.9167 & -1.5556 & 21.7778 \\
5 & .1111 & -.2857 & -.2857 & 1.0000 & 1.0000 & -6.2222 & -6.2222 \\
6 & .1944 & -.1429 & -.6429 & .0000 & 2.7500 & 3.1111 & -20.2222 \\
7 & .2778 & .1190 & -.6032 & -1.3889 & -.1667 & 7.7778 & 31.1111 \\
8 & .3611 & .5000 & .1111 & -1.3889 & -4.7500 & -10.8889 & -20.8889 \\
9 & .4444 & 1.0000 & 1.7778 & 2.7778 & 4.0000 & 5.4444 & 7.1111 \\
\hline
\end{tabular}

TABLE 9

Values of $c_{i}$ for $n=10$

\begin{tabular}{|r|c|r|r|r|r|r|r|}
\hline $\mathrm{i}$ & $\mathrm{k}=1$ & $\mathrm{k}=2$ & $\mathrm{k}=3$ & $\mathrm{k}=4$ & $\mathrm{k}=5$ & \multicolumn{1}{c|}{$\mathrm{k}=6$} & \multicolumn{1}{c|}{$\mathrm{k}=7$} \\
\hline 1 & -.2000 & .3000 & -.4000 & .5000 & -.6000 & .7000 & -.8000 \\
2 & -.1333 & .0333 & .2667 & -.8333 & 1.7333 & -3.0333 & 4.8000 \\
3 & -.0667 & -.1500 & .4333 & -.4167 & -.6000 & 3.7333 & -10.6000 \\
4 & .0000 & -.2500 & .2667 & .4167 & -1.6000 & 1.0000 & 8.0000 \\
5 & .0667 & -.2667 & -.0667 & .8333 & -.2667 & -3.7333 & 5.6000 \\
6 & .1333 & -.2000 & -.4000 & .5000 & 1.6000 & -1.8667 & -11.2000 \\
7 & .2000 & -.0500 & -.5667 & -.4167 & 1.6000 & 4.2000 & -2.8000 \\
8 & .2667 & .1833 & -.4000 & -1.2500 & -1.0667 & 3.2667 & 17.6000 \\
9 & .3333 & .5000 & .2667 & -.8333 & -3.4000 & -8.1667 & -16.0000 \\
10 & .4000 & .9000 & 1.6000 & 2.5000 & 3.6000 & 4.9000 & 6.4000 \\
\hline
\end{tabular}


TABLE 10

Values of $c_{i}$ for $n=11$

\begin{tabular}{|r|c|c|c|r|r|r|r|}
\hline $\mathrm{i}$ & $\mathrm{k}=1$ & $\mathrm{k}=2$ & $\mathrm{k}=3$ & $\mathrm{k}=4$ & $\mathrm{k}=5$ & \multicolumn{1}{c|}{$\mathrm{k}=6$} & \multicolumn{1}{c|}{$\mathrm{k}=7$} \\
\hline 1 & -.1818 & .2727 & -.3636 & .4545 & -.5455 & .6364 & -.7273 \\
2 & -.1273 & .0545 & .1818 & -.6364 & 1.3636 & -2.4182 & 3.8545 \\
3 & -.0727 & -.1030 & .3636 & -.4545 & -.1212 & 2.1636 & -6.8364 \\
4 & -.0182 & -.2000 & .2879 & .1515 & -1.1818 & 1.6545 & 2.2000 \\
5 & .0364 & -.2364 & .0606 & .6061 & -.7273 & -1.6727 & 5.9636 \\
6 & .0909 & -.2121 & -.2121 & .6061 & .6061 & -2.5455 & -2.5455 \\
7 & .1455 & -.1273 & -.4242 & .1212 & 1.4545 & .5091 & -7.1273 \\
8 & .2000 & .0182 & -.4697 & -.6061 & .7273 & 3.5636 & 3.0182 \\
9 & .2545 & .2242 & -.2424 & -1.0606 & -1.3939 & .8909 & 9.8909 \\
10 & .3091 & .4909 & .3636 & -.4545 & -2.4545 & -6.2364 & -12.5091 \\
11 & .3636 & .8182 & 1.4545 & 2.2727 & 3.2727 & 4.4545 & 5.8182 \\
\hline
\end{tabular}

TABLE 11

Values of $c_{i}$ for $n=12$

\begin{tabular}{|r|c|c|r|r|r|r|r|}
\hline $\mathrm{i}$ & $\mathrm{k}=1$ & $\mathrm{k}=2$ & $\mathrm{k}=3$ & $\mathrm{k}=4$ & $\mathrm{k}=5$ & $\mathrm{k}=6$ & \multicolumn{1}{c|}{$\mathrm{k}=7$} \\
\hline 1 & -.1667 & .2500 & -.3333 & .4167 & -.5000 & .5833 & -.6667 \\
2 & -.1212 & .0682 & .1212 & -.4924 & 1.0909 & -1.9621 & 3.1515 \\
3 & -.0758 & -.0682 & .3030 & -.4470 & .1364 & 1.2197 & -4.4848 \\
4 & -.0303 & -.1591 & .2828 & -.0126 & -.8182 & 1.6439 & -.2424 \\
5 & .0152 & -.2045 & .1313 & .4040 & -.8182 & -.4242 & 4.2121 \\
6 & .0606 & -.2045 & -.0808 & .5556 & .0000 & -1.9697 & 1.2121 \\
7 & .1061 & -.1591 & -.2828 & .3535 & .9091 & -1.0606 & -4.2424 \\
8 & .1515 & -.0682 & -.4040 & -.1313 & 1.0909 & 1.4848 & -3.1515 \\
9 & .1970 & .0682 & -.3737 & -.6692 & .1364 & 2.5985 & 4.4848 \\
10 & .2424 & .2500 & -.1212 & -.8712 & -1.4545 & -.3712 & 5.3333 \\
11 & .2879 & .4773 & .4242 & -.1894 & -1.7727 & -4.8258 & -9.9394 \\
12 & .3333 & .7500 & 1.3333 & 2.0833 & 3.0000 & 4.0833 & 5.3333 \\
\hline
\end{tabular}

TABLE 12

Values of $c_{i}$ for $n=13$

\begin{tabular}{|r|c|c|r|r|r|r|r|}
\hline $\mathrm{i}$ & $\mathrm{k}=1$ & $\mathrm{k}=2$ & $\mathrm{k}=3$ & $\mathrm{k}=4$ & $\mathrm{k}=5$ & $\mathrm{k}=6$ & \multicolumn{1}{c|}{$\mathrm{k}=7$} \\
\hline 1 & -.1538 & .2308 & -.3077 & .3846 & -.4615 & .5385 & -.6154 \\
2 & -.1154 & .0769 & .0769 & -.3846 & .8846 & -1.6154 & 2.6154 \\
3 & -.0769 & -.0420 & .2517 & -.4196 & .2727 & .6364 & -2.9650 \\
4 & -.0385 & -.1259 & .2657 & -.1119 & -.5350 & 1.4196 & -1.2028 \\
5 & .0000 & -.1748 & .1678 & .2448 & -.7552 & .2448 & 2.5175 \\
6 & .0385 & -.1888 & .0070 & .4545 & -.3147 & -1.2238 & 2.2727 \\
7 & .0769 & -.1678 & -.1678 & .4196 & .4196 & -1.3986 & -1.3986 \\
8 & .1154 & -.1119 & -.3077 & .1399 & .9091 & .0000 & -3.4965 \\
9 & .1538 & -.0210 & -.3636 & -.2867 & .7133 & 1.7133 & -.5594 \\
10 & .1923 & .1049 & -.2867 & -.6643 & -.2413 & 1.7133 & 4.3357 \\
11 & .2308 & .2657 & -.0280 & -.6993 & -1.3916 & -1.0280 & 2.5734 \\
12 & .2692 & .4615 & .4615 & .0000 & -1.2692 & -3.7692 & -8.0000 \\
13 & .03077 & .6923 & 1.2308 & 1.9231 & 2.7692 & 3.7692 & 4.9231 \\
\hline
\end{tabular}


TABLE 13

Values of $c_{i}$ for $n=14$

\begin{tabular}{|r|c|c|r|r|r|r|r|}
\hline $\mathrm{i}$ & $\mathrm{k}=1$ & $\mathrm{k}=2$ & $\mathrm{k}=3$ & $\mathrm{k}=4$ & \multicolumn{1}{c|}{$\mathrm{k}=5$} & $\mathrm{k}=6$ & \multicolumn{1}{c|}{$\mathrm{k}=7$} \\
\hline $\mathrm{l}$ & -.1429 & .2143 & -.2857 & .3571 & -.4286 & .5000 & -.5714 \\
2 & -.1099 & .0824 & .0440 & -.3022 & .7253 & -1.3462 & 2.1978 \\
3 & -.0769 & -.0220 & .2088 & -.3846 & .3407 & .2692 & -1.9560 \\
4 & -.0440 & -.0989 & .2438 & -.1698 & -.3237 & 1.1503 & -1.4945 \\
5 & -.0110 & -.1484 & .1838 & .1254 & -.6384 & .5629 & 1.2747 \\
6 & .0220 & -.1703 & .0639 & .3472 & -.4496 & -.6119 & 2.1978 \\
7 & .0549 & -.1648 & -.0809 & .4046 & .0749 & -1.2238 & .2747 \\
8 & .0879 & -.1319 & -.2158 & .2697 & .5994 & -.6993 & -2.1978 \\
9 & .1209 & -.0714 & -.3057 & -.0225 & .7742 & .6119 & -2.1978 \\
10 & .1538 & .0165 & -.3157 & -.3741 & .3896 & 1.5909 & .8791 \\
11 & .1868 & .1319 & -.2108 & -.6244 & -.4705 & 1.0035 & 3.6484 \\
12 & .2198 & .2747 & .0440 & -.5495 & -1.2747 & -1.3462 & .8791 \\
13 & .2527 & .4451 & .4835 & .1374 & -.8901 & -2.9615 & -6.5055 \\
14 & .2857 & .6429 & 1.1429 & 1.7857 & 2.5714 & 3.5000 & 4.5714 \\
\hline
\end{tabular}

TABLE 14

Values of $c_{i}$ for $n=15$

\begin{tabular}{|r|c|c|r|r|r|r|r|}
\hline $\mathrm{i}$ & $\mathrm{k}=1$ & $\mathrm{k}=2$ & $\mathrm{k}=3$ & $\mathrm{k}=4$ & $\mathrm{k}=5$ & $\mathrm{k}=6$ & \multicolumn{1}{c|}{$\mathrm{k}=7$} \\
\hline 1 & -.1333 & .2000 & -.2667 & .3333 & -.4000 & .4667 & -.5333 \\
2 & -.1048 & .0857 & .0190 & -.2381 & .6000 & -1.1333 & 1.8667 \\
3 & -.0762 & -.0066 & .1729 & -.3480 & .3692 & .0359 & -1.2718 \\
4 & -.0476 & -.0769 & .2205 & -.2015 & -.1692 & .8974 & -1.4872 \\
5 & -.0190 & -.1253 & .1875 & .0383 & -.5119 & .6821 & .4513 \\
6 & .0095 & -.1516 & .0996 & .2501 & -.4825 & -.1795 & 1.7436 \\
7 & .0381 & -.1560 & -.0176 & .3546 & -.1399 & -.8974 & 1.0256 \\
8 & .0667 & -.1385 & -.1385 & .3147 & .3147 & -.8974 & -.8974 \\
9 & .0952 & -.0989 & -.2374 & .1349 & .6294 & -.1282 & -2.0513 \\
10 & .1238 & -.0374 & -.2886 & -.1382 & .5944 & .8974 & -1.0256 \\
11 & .1524 & .0462 & -.2667 & -.4159 & .1343 & 1.3282 & 1.5590 \\
12 & .1810 & .1516 & -.1458 & -.5678 & -.6000 & .4667 & 2.8513 \\
13 & .2095 & .2791 & .0996 & -.4212 & -1.1385 & -1.4718 & -.1641 \\
14 & .2381 & .4286 & .4952 & .2381 & -.6000 & -2.3333 & -5.3333 \\
15 & .2667 & .6000 & 1.0667 & 1.6667 & 2.4000 & 3.2667 & 4.2667 \\
\hline
\end{tabular}

TABLE 15

Values of $c_{i}$ for $n=16$

\begin{tabular}{|r|c|c|r|r|r|r|r|}
\hline $\mathrm{i}$ & $\mathrm{k}=1$ & $\mathrm{k}=2$ & $\mathrm{k}=3$ & $\mathrm{k}=4$ & $\mathrm{k}=5$ & $\mathrm{k}=6$ & $\mathrm{k}=7$ \\
\hline $\mathrm{l}$ & -.1250 & .1875 & -.2500 & .3125 & -.3750 &. .4375 & -.5000 \\
2 & -.1000 & .0875 & .0000 & -.1875 & .5000 & -.9625 & 1.6000 \\
3 & -.0750 & .0054 & .1429 & -.3125 & .3750 &.- .1125 & -.8000 \\
4 & -.0500 & -.0589 & .1978 & -.2163 & -.0577 & .6798 & -1.3538 \\
5 & -.0250 & -.1054 & .1841 & -.0240 & -.3942 & .6933 & -.0615 \\
6 & .0000 & -.1339 & .1209 & .1683 & -.4615 & .1010 & 1.2308 \\
7 & .0250 & -.1446 & .0275 & .2933 & -.2596 & -.5721 & 1.2308 \\
8 & .0500 & -.1375 & -.0769 & .3125 & .0962 & -.8413 & .0000 \\
9 & .0750 & -.1125 & -.1731 & .2163 & .4327 & -.5048 & -1.3462 \\
10 & .1000 & -.0696 & -.2418 & .0240 & .5769 & .2644 & -1.5385 \\
11 & .1250 & -.0089 & -.2637 & -.2163 & .4135 & .9760 & -.1538 \\
12 & .1500 & .0696 & -.2198 & -.4279 & -.0577 & 1.0298 & 1.7846 \\
13 & .1750 & .1661 & -.0907 & -.5048 & -.6635 & .0740 & 2.1077 \\
14 & .2000 & .2804 & .1429 & -.3125 & -1.0000 & -1.4875 & -.8000 \\
15 & .2250 & .4125 & .5000 & .3125 & -.3750 & -1.8375 & -4.4000 \\
16 & .2500 & .5625 & 1.0000 & 1.5625 & 2.2500 & 3.0625 & 4.0000 \\
\hline
\end{tabular}


TABLE 16

Values of $c_{i}$ for $n=17$

\begin{tabular}{|r|c|c|c|r|r|r|r|}
\hline $\mathrm{i}$ & $\mathrm{k}=1$ & $\mathrm{k}=2$ & $\mathrm{k}=3$ & $\mathrm{k}=4$ & $\mathrm{k}=5$ & \multicolumn{1}{c|}{$\mathrm{k}=6$} & \multicolumn{1}{c|}{$\mathrm{k}=7$} \\
\hline $\mathrm{l}$ & -.1176 & .1765 & -.2353 & .2941 & -.3529 & .4118 & -.4706 \\
2 & -.0956 & .0882 & -.0147 & -.1471 & .4191 & -.8235 & 1.3824 \\
3 & -.0735 & .0147 & .1176 & -.2794 & .3676 & -.2059 & -.4706 \\
4 & -.0515 & -.0441 & .1765 & -.2206 & .0221 & .5000 & -1.1765 \\
5 & -.0294 & -.0882 & .1765 & -.0679 & -.2919 & .6493 & -.3620 \\
6 & -.0074 & -.1176 & .1324 & .1018 & -.4146 & .2692 & .7783 \\
7 & .0147 & -.1324 & .0588 & .2319 & -.3156 & -.3009 & 1.1584 \\
8 & .0368 & -.1324 & -.0294 & .2862 & -.0560 & -.6810 & .5068 \\
9 & .0588 & -.1176 & -.1176 & .2489 & .2489 & -.6335 & -.6335 \\
10 & .0809 & -.0882 & -.1912 & .1244 & .4666 & -.1584 & -1.3462 \\
11 & .1029 & -.0441 & -.2353 & -.0622 & .4853 & .5000 & -.9593 \\
12 & .1250 & .0147 & -.2353 & -.2658 & .2506 & .9344 & .4253 \\
13 & .1471 & .0882 & -.1765 & -.4208 & -.1968 & .7443 & 1.7557 \\
14 & .1691 & .1765 & -.0441 & -.4412 & -.6838 & -.2059 & 1.4706 \\
15 & .1912 & .2794 & .1765 & -.2206 & -.8676 & -1.4412 & -1.1765 \\
16 & .2132 & .3971 & .5000 & .3676 & -.1985 & -1.4412 & -3.6471 \\
17 & .2353 & .5294 & .9412 & 1.4706 & 2.1176 & 2.8824 & 3.7647 \\
\hline
\end{tabular}

TABLE 17

Values of $c_{i}$ for $n=18$

\begin{tabular}{|r|c|c|c|r|r|r|r|}
\hline $\mathrm{i}$ & $\mathrm{k}=1$ & $\mathrm{k}=2$ & $\mathrm{k}=3$ & $\mathrm{k}=4$ & $\mathrm{k}=5$ & $\mathrm{k}=6$ & $\mathrm{k}=7$ \\
\hline $\mathrm{l}$ & -.1111 & .1667 & -.2222 & .2778 & -.3333 & .3889 & -.4444 \\
2 & -.0915 & .0882 & -.0261 & -.1144 & .3529 & -.7092 & 1.2026 \\
3 & -.0719 & .0221 & .0964 & -.2492 & .3529 & -.2631 & -.2386 \\
4 & -.0523 & -.0319 & .1569 & -.2181 & .0784 & .3546 & -.9935 \\
5 & -.0327 & -.0735 & .1667 & -.0980 & -.2059 & .5801 & -.5229 \\
6 & -.0131 & -.1029 & .1373 & .0490 & -.3575 & .3599 & .4183 \\
7 & .0065 & -.1201 & .0801 & .1757 & -.3311 & -.0941 & .9673 \\
8 & .0261 & -.1250 & .0065 & .2492 & -.1538 & -.4953 & .7320 \\
9 & .0458 & -.1176 & -.0719 & .2516 & .0995 & -.6194 & -.0915 \\
10 & .0654 & -.0980 & -.1438 & .1797 & .3318 & -.3871 & -.9150 \\
11 & .0850 & -.0662 & -.1977 & .0449 & .4480 & .1065 & -1.1209 \\
12 & .1046 & -.0221 & -.2222 & -.1266 & .3801 & .6170 & -.4444 \\
13 & .1242 & .0343 & -.2059 & -.2941 & .1124 & .8297 & .7712 \\
14 & .1438 & .1029 & -.1373 & -.4020 & -.2941 & .4918 & 1.5948 \\
15 & .1634 & .1838 & -.0049 & -.3799 & -.6765 & -.4003 & .94747 \\
16 & .1830 & .2770 & .2026 & -.1430 & -.7451 & -1.3611 & -1.3856 \\
17 & .2026 & .3824 & .4967 & .4085 & -.0588 & -1.1209 & -3.0327 \\
18 & .2222 & .5000 & .8889 & 1.3889 & 2.0000 & 2.7222 & 3.5556 \\
\hline
\end{tabular}


TABLE 18

Values of $c_{i}$ for $n=19$

\begin{tabular}{|r|c|r|r|r|r|r|r|}
\hline $\mathrm{i}$ & $\mathrm{k}=1$ & $\mathrm{k}=2$ & $\mathrm{k}=3$ & $\mathrm{k}=4$ & $\mathrm{k}=5$ & $\mathrm{k}=6$ & \multicolumn{1}{c|}{$\mathrm{k}=7$} \\
\hline 1 & -.1053 & .1579 & -.2105 & .2632 & -.3158 & .3684 & -.4211 \\
2 & -.0877 & .0877 & -.0351 & -.0877 & .2982 & -.6140 & 1.0526 \\
3 & -.0702 & .0279 & .0784 & -.2219 & .3344 & -.2962 & -.0743 \\
4 & -.0526 & -.0217 & .1391 & -.2116 & .1176 & .2384 & -.8220 \\
5 & -.0351 & -.0609 & .1558 & -.1182 & -.1352 & .5021 & -.5944 \\
6 & -.0175 & -.0898 & .1378 & .0077 & -.2988 & .3989 & .1486 \\
7 & .0000 & -.1084 & .0939 & .1264 & -.3220 & .0542 & .7430 \\
8 & .0175 & -.1166 & .0333 & .2090 & -.2110 & -.3215 & .7802 \\
9 & .0351 & -.1146 & -.0351 & .2374 & -.0124 & -.5346 & .2601 \\
10 & .0526 & -.1022 & -.1022 & .2043 & .2043 & -.4768 & -.4768 \\
11 & .0702 & -.0795 & -.1589 & .1135 & .3633 & -.1589 & -.9536 \\
12 & .0877 & -.0464 & -.1963 & -.0206 & .3994 & .2890 & -.8127 \\
13 & .1053 & -.0031 & -.2054 & -.1729 & .2755 & .6517 & -.0372 \\
14 & .1228 & .0506 & -.1770 & -.3070 & -.0005 & .6971 & .9474 \\
15 & .1404 & .1146 & -.1022 & -.3762 & -.3591 & .2781 & 1.3746 \\
16 & .1579 & .1889 & .0281 & -.3225 & -.6517 & -.5310 & .5294 \\
17 & .1754 & .2735 & .2229 & -.0774 & -.6336 & -1.2642 & -1.4861 \\
18 & .1930 & .3684 & .4912 & .4386 & .0526 & -.8596 & -2.5263 \\
19 & .2105 & .4737 & .8421 & 1.3158 & 1.8947 & 2.5789 & 3.3684 \\
\hline
\end{tabular}

TABLE 19

Values of $c_{i}$ for $n=20$

\begin{tabular}{|r|c|c|r|r|r|r|r|}
\hline $\mathrm{i}$ & $\mathrm{k}=1$ & $\mathrm{k}=2$ & $\mathrm{k}=3$ & $\mathrm{k}=4$ & $\mathrm{k}=5$ & $\mathrm{k}=6$ & $\mathrm{k}=7$ \\
\hline 1 & -.1000 & .1500 & -.2000 & .2500 & -.3000 & .3500 & -.4000 \\
2 & -.0842 & .0868 & -.0421 & -.0658 & .2526 & -.5342 & .9263 \\
3 & -.0684 & .0325 & .0632 & -.1974 & .3140 & -.3132 & .0421 \\
4 & -.0526 & -.0132 & .1230 & -.2025 & .1443 & .1463 & -.6687 \\
5 & -.0368 & -.0500 & .1447 & -.1309 & -.0779 & .4242 & -.6102 \\
6 & -.0211 & -.0781 & .1354 & -.0241 & -.2429 & .4047 & -.0446 \\
7 & -.0053 & -.0974 & .1024 & .0845 & -.2989 & .1549 & .5276 \\
8 & .0105 & -.1079 & .0528 & .1696 & -.2393 & -.1739 & .7282 \\
9 & .0263 & -.1096 & -.0060 & .2141 & -.0912 & -.4226 & .4551 \\
10 & .0421 & -.1026 & -.0669 & .2090 & .0966 & -.4789 & -.1214 \\
11 & .0579 & -.0868 & -.1226 & .1533 & .2656 & -.3099 & -.6675 \\
12 & .0737 & -.0623 & -.1659 & .0542 & .3595 & .0282 & -.8495 \\
13 & .0895 & -.0289 & -.1897 & -.0729 & .3361 & .4015 & -.5006 \\
14 & .1053 & .0132 & -.1866 & -.2045 & .1789 & .6327 & .2601 \\
15 & .1211 & .0640 & -.1494 & -.3090 & -.0901 & .5575 & 1.0068 \\
16 & .1368 & .1237 & -.0710 & -.3466 & -.3997 & .1024 & 1.1368 \\
17 & .1526 & .1921 & .0559 & -.2696 & -.6164 & -.6144 & .2006 \\
18 & .1684 & .2693 & .2386 & -.0219 & -.5333 & -1.1605 & -1.5158 \\
19 & .1842 & .3553 & .4842 & .4605 & .1421 & -.6447 & -2.1053 \\
20 & .2000 & .4500 & .8000 & 1.2500 & 1.8000 & 2.4500 & 3.2000 \\
\hline
\end{tabular}


Retired from Office of Naval Research

2630 Kingsbridge Terrace

Bronx, New York 10463

Department of Mathematics and Computer Science

Herbert H. Lehman College

City University of New York

Bronx, New York 10468

1. A. Fletcher, J. C. P. Miller, L. Rosenhead \& L. J. Comrie, An Index of Mathematical Tables, Vol. 1, 2nd ed., Addison-Wesley, Reading, Mass., 1962, pp. 594-596.

2. JACK LADERMAN, "The square root method for solving simultaneous linear equations," MTAC, v. 3 , 1948, pp. 13-16.

3. JAMES Singer, Elements of Numerical Analysis, Academic Press, New York, 1964, p. 99. 